\title{
La planète Bidonville. Le cas des Chabolas de Madrid
}

Joanne Rasse et Paul Rasse

\section{(2) OpenEdition}

Édition électronique

URL : http://journals.openedition.org/communicationorganisation/356

DOI : 10.4000/communicationorganisation.356

ISSN : $1775-3546$

Éditeur

Presses universitaires de Bordeaux

\section{Édition imprimée}

Date de publication : 1 décembre 2007

Pagination : 106-121

ISSN : 1168-5549

\section{Référence électronique}

Joanne Rasse et Paul Rasse, "La planète Bidonville. Le cas des Chabolas de Madrid ", Communication et organisation [En ligne], 32 | 2007, mis en ligne le 01 décembre 2010, consulté le 30 avril 2019. URL: http://journals.openedition.org/communicationorganisation/356 ; DOI : 10.4000/

communicationorganisation.356 
Dossier : La ville dans tous les sens

\title{
Résumé
}

Les quartiers des centre villes en cours de gentrification, tout de circulation, de communication et de métissage, s'opposent, par bien des aspects, symétriquement aux bidonvilles, où viennent s'agglutiner, se sédimenter les populations récemment urbanisées, abandonnées à elles-mêmes, dans une urbanité informelle en marge de la cité institutionnelle. Pour leur croissance incontrôlable et leur précarité propice aux mafias et au communautarisme, ils constituent, en dépit des ressources d'inventivité qu'ils mobilisent, un des grands maux de notre époque. Une enquête urbanistique menée sur les Chabolas, les bidonvilles de Madrid nous permet d'en prendre la mesure. Nous conjuguerons la description de cette microsociété avec une mise en perspective des connaissances recueillies en les resituant dans un projet anthropologique plus global, d'analyse de la civilisation planétaire, tissée par les réseaux de communication dont sont exclus les population des bidonvilles.

\section{Mots-clés}

Bidonvilles, fluidité/immobilisme des populations, métissage/communautarisme, anthropologie de la communication

\begin{abstract}
The districts of the town centres, which are on the way to gentrification, characterized by movement, communication and racial mixture, contrast significantly with the shanty towns where recently urbanized populations stick together and settle down, left on their own in an unorganized urbanity on the fringe of the institutional city. Because of their uncontrollable growth and their precariousness, and thus more vulnerable to mafia and group building, they constitute, despite their inventiveness, one of the major issues of our time. An urban inquiry made on the Chabolas, the shanty towns of Madrid, enables to measure their extent. We will combine the description of this micro society with the knowledge gathered, by resetting it in a more global anthropological project of analysis of the world's civilization, woven by the communication networks from which shanty town populations are excluded.
\end{abstract}

\section{Key-Words}

Shanty town, interbreeding, communautarism, anthropology of communication

Paul Rasse est Professeur des Universités, directeur du Laboratoire de recherche en Sciences de l'Information et de la Communication I3M (Information, Milieux, Médias, Médiation) - Université de Nice-Sophia Antipolis. Joanne Rasse est architecte, (ENSAPB) École Nationale Supérieure d'Architecture de Paris-Belleville. 


\title{
La planète Bidonville. Le cas des Chabolas de Madrid
}

Joanne Rasse et Paul Rasse

\author{
rasse@unice.fr \\ rassejoanne@hotmail.fr
}

Alors qu'au début du XIX ${ }^{\mathrm{e}}$ siècle, $98 \%$ de l'humanité habitait à la campagne, à l'aube de ce que l'ONU appelle " le millénaire urbain », la moitié de la population mondiale vit aujourd'hui dans les villes, et ce chiffre augmente chaque jour de 180000 personnes ${ }^{1}$. Cette progression est principalement imputable aux pays les moins développés, pour l'instant encore essentiellement ruraux, et pour lesquels elle atteint en moyenne $2,3 \%$ par an ; si bien que la population urbaine devrait encore doubler d'ici une trentaine d'années. Or la ville a bien du mal à absorber l'augmentation rapide de cette population, qui entraîne une urbanité informelle, au sens de « non officielle», en marge de la cité institutionnelle.

Les bidonvilles se développent au fur et à mesure que les zones rurales se désertifient, que l'essor des moyens de transport ruine les systèmes agricoles anciens, autarciques, qui nourrissaient et retenaient les populations rurales, tandis que les médias ouvrent les sociétés traditionnelles à de nouveaux univers, où la ville fait figure d'eldorado. L'involution urbaine répond à l'exode rural en servant de déversoir à une main d'œuvre devenue excédentaire. Attirée par les lumières de la ville, celle-ci vient grossir les zones les plus pauvres, au prix de systèmes d'auto-exploitation et de subdivision accrue de niches d'activités et de logement, déjà occupés de manière très dense. Le phénomène n'est pas nouveau. En Occident, il s'est produit au $\mathrm{XIX}^{\mathrm{e}}$ siècle, à la suite de la première grande révolution des moyens de communication, alors que la ville et les nouveaux centres industriels attiraient une main d'œuvre immigrée venues des campagnes proches et lointaines, elles aussi en mutation. Mais le problème actuel est d'une tout autre ampleur et devient planétaire. Comme toujours, il est difficile d'avoir des chiffres précis, dès qu'ils concernent les populations les plus pauvres. Cependant les experts de l'ONU

\footnotetext{
${ }^{1}$ Le millénaire Urbain, rapport pour la session extraordinaire de l'assemblée générale des Nations Unies consacrée à l'examen et à l'évaluation d'ensemble de l'application du programme pour l'habitat, New York, 6 - 8 juin 2001. Voir aussi : Davis Mike, «De l'explosion urbaine au bidonville global, Les petits sorciers de Kinshasa », Le Monde diplomatique du 6 septembre 2006.
} 
évaluent à un milliard, soit un habitant de la planète sur six, le nombre de personnes qui vivent déjà dans des bidonvilles et prévoient que ces chiffres doubleront d'ici $2030^{2}$. Mike Davis recense 250000 bidonvilles sur la planète, les 5 plus grandes métropoles d'Asie, ajoute-t-il, (Karachi, Bombay, Delhi, Calcutta, Dhaka) comptent à elles seules 15000 bidonvilles distincts, avec une population totale de plus de 20 millions de personnes. Et d'énumérer ainsi les Bustees de Calcutta, les Kampungs de Djakarta, les Iskwaters de Manille, les Shammasas de Khartoum, les Umjondolos de Durban, les Intra-muros de Rabat, les Bidonvilles d'Abidjan, les Baladis du Caire, les Gecekondus d'Ankara, les Conventillos de Quito, les Favelas du Brésil, les Villas miserias de Buenos Aires, les Colonias populares de Mexico (...) $)^{3}$. Dans la même veine, nous y ajouterons les Chabolas en Espagne qui constituent le terrain sur lequel nous avons pu nous pencher. Et Mike Davis de conclure ainsi : "seul le bidonville demeure une solution totalement admise au problème de stockage de l'humanité excédentaire du $\mathrm{XXI}^{\mathrm{e}}$ siècle. ${ }^{4} \gg$ Cette situation alarmiste met en question l'habitat et la sociabilité des villes de demain. Alors que les solutions appliquées jusque-là pour résoudre la misère (croissance, consommation et Welfare State) semblent s'essouffler.

Nous nous proposons de rendre compte d'une enquête urbanistique menée sur les Chabolas, les bidonvilles de Madrid's. Nous conjuguerons la description d'une microsociété et une mise en perspective des connaissances recueillies, en les resituant dans un projet anthropologique global d'analyse de la civilisation dans ses aspects planétaires ${ }^{6}$. En effet, comment saisir ces mutations du monde, sans se noyer dans des approches trop générales et superficielles des phénomènes étudiés qui conduisent trop facilement à des banalités ? Et en même temps l'immersion lente dans un milieu, l'observation patiente d'un objet ne risquent-elles pas de nous faire perdre l'essentiel, que le monde se transforme et qu'il n'a plus de sens que

\footnotetext{
${ }^{2}$ Davis Mike, Planète Bidonvilles, Bruno Bachmann, Les enfants d'une même agonie, Ab Irato, Paris, 2005, p.22.

${ }^{3}$ Idem

${ }^{4}$ Idem, p.42.

${ }^{5}$ La recherche sur le terrain a été principalement menée par Joanne Rasse et Nicolas Gueniau en 2003 à Madrid, pour l'école d'Architecture de Paris Belleville.

${ }^{6}$ Le matériau accumulé par l'enquête de terrain a été repris dans la perspective de travaux récents sur la globalisation. Cf notamment: Rasse Paul, La rencontre des mondes, Diversité culturelle et communication, Armand Colin, 2006 ; et aussi Rasse Paul, «L'anthropologie de la communication pour étudier la rencontre des mondes », in Communication interculturelle et diversité en Méditerranée, sous la direction de Françoise Albertini, Éditions Dumane, 2006.
} 
par le mouvement, ou même mieux par l'accélération d'un mouvement globalisé, où tout est en interdépendance? On ne peut plus se contenter d'étudier, ici et maintenant, des sociétés en mutation, en faisant l'impasse sur leur évolution et sur les forces qui les transforment à vitesse accélérée. Comme le précise Marc Auger, « s'adapter au changement d'échelle, ce n'est pas cesser de privilégier l'observation de petites unités, mais prendre en considération les mondes qui les traversent, les débordent, et ce faisant, ne cessent de les constituer et de les reconstituer $\gg$. Aussi le cas des Chabolas nous a paru un bel objet anthropologique pour saisir la relégation des populations récemment urbanisées, en opposition aux nouveaux usages de la ville, tout en fluidité, tels qu'en profitent les catégories sociales les plus aisées. Comment les bidonvilles et plus particulièrement les Chabolas expriment-ils la dynamique des populations qu'ils abritent (leur histoire, les contraintes auxquelles elles sont confrontées, leurs modes de vie pratiques, quotidiens) ? Comment les limites qui dessinent les relations entre « soi et l'autre " structurent-elles l'espace et la sociabilité des personnes qui habitent ces territoires? Enfin comment ce mouvement est-il significatif de mutations profondes qui travaillent la société bousculée par la révolution des moyens de communication?

\section{L'enclave d'El Salobral, bidonville gitan, au sud de Madrid.}

Le terme «Chabola» est utilisé en Espagne pour désigner les bidonvilles, qui s'implantent dans les lieux résiduels résultant de l'urbanisme rapide des banlieues, lorsque des espaces délaissés ont illégalement été appropriés par une population démunie. Le bidonville étudié, «El Salobral», est caractérisé par son ancienneté (une vingtaine d'années), sa communauté homogène (composée à $80 \%$ de gitans sédentarisés, ou en voie de sédentarisation), et par sa taille (approximativement 20 hectares, occupés par plus de 400 familles). En dépit de tentatives de relogement, sa croissance est devenue, à l'heure actuelle, quasiment incontrôlable.

La Chabola El Salobral se situe au delà de la périphérie nord-est, non consolidée, de Madrid et dessine les «limites» actuelles de la ville officielle. La route reliant le sud de l'Espagne à la capitale, longe sur plus de deux kilomètres un terrain cahoteux, où seule apparaît une mer de toits de tôle soutenue par des amas de pneus ou de pierres... Le bidonville est encerclé par la ligne de chemin de fer Madrid-Alicante,

\footnotetext{
7 Augé Marc, Pour une anthropologie des mondes contemporains, Champs Flammarion, 2001, p.178.
} 
par les routes périphériques M40 et M30, et enfin par l'avenue de l'Andalousie. De fait, le bidonville s'est glissé dans les interstices délaissés des grands réseaux de communication, comme cela est fréquent partout dans le monde. Ces abords des axes de circulation, interrompus, infranchissables, autant qu'intenses, bruyants, polluants, comme les voies ferrées, les autoroutes, sont impropres à la promotion immobilière. Ils deviennent des lieux de prédilection pour l'installation des bidonvilles. Les grands axes de transport sont à la fois des éléments de connexions et de fragmentation du territoire, ils constituent les barrières modernes et artificielles de cet habitat, que paradoxalement ils isolent et rendent difficile d'accès. C'est d'ailleurs le statut d'enclave d'«El Salobral», à l'intérieur de l'ensemble urbain, qui explique et garantit sa longévité. Autre conséquence de cet état de fait, la Chabola, comme la plupart des bidonvilles, est strictement bornée et entourée de limites physiques inamovibles, qui la contiennent et l'obligent à grossir par densification, ce qui conduit à une dégradation continuelle des conditions de vie, de promiscuité et de salubrité. ${ }^{8}$

Ce qui définit un bidonville, précise Backman «ce n'est pas tant le mot bidon -sa matière- mais l'illégalité de son implantation $»{ }^{9}$ En effet explique-t-il, certains d'entre eux, comme les favelas Brésiliennes sont construits en dur, mais illégalement, sur des espaces publics ou privés qui n'appartiennent pas aux habitants et les obligent, depuis la première pierre, à la plus grande précarité ; à commencer par leur ôter tout droit formel, par les soumettre, dans un rapport quasi féodal, au bon vouloir des administrations, des fonctionnaires, des politiques et des polices qui les tolèrent.

La Chabola El Salobral suit le même processus. Au départ, dans les années 1980, le terrain en friche a été squatté par les habitants de San Cristobal de Los Angeles, quartier populaire de Madrid, où fut relogée une partie de la population des bidonvilles des années 50, pour y établir des jardins ouvriers; les cultures potagères leur permettant d'améliorer leur quotidien. Très vite, de petites cahutes y ont été construites pour abriter l'outillage, et éventuellement les jardiniers d'occasion. Quelques années plus tard, les premières familles gitanes ont commencé à s'y installer et à demander aux habitants de San Cristobal d'acheter les cabanes pour les habiter, ces transactions, sans doute pour des sommes modestes, mais en toute illégalité, ne leur

\footnotetext{
${ }^{8}$ Davis Mike, Planète Bidonvilles, Bachmann Bruno, Les enfants d'une même agonie, Ab Irato, Paris, 2005, p.23.

${ }^{9}$ Idem. p.60.
} 
donnaient aucun droit, puisque le vendeur n'était propriétaire de rien. Avec le non statut du sol, le lieu, comme celui de la plupart des bidonvilles, a basculé dans l'illégalité au fur et à mesure que les baraques y étaient construites. Dès lors, les habitants ne pouvaient plus revendiquer un droit d'accès aux infrastructures officielles, réseaux d'eaux, d'assainissement, d'électricité, de transports.

Bien que l'aspect chaotique puisse donner l'impression d'un environnement déconstruit, le bidonville a une logique propre d'attribution, de constitution et de partage de l'espace, dans le respect d'un minimum de règles tacites, informelles, que rappellent et interprètent les gens de pouvoir (le père, les chefs de clan, de quartier ou de rue, les mafias...). Les "règles orales» de la communauté gitane, sourdes, latentes, mais toujours présentes administrent la Chabola. Chacun a son espace, sa place, son rôle. La hiérarchie est dictée par la communauté et le respect des ancêtres. L'absence de structure urbaine et d'institution est relayée par une structure communautaire très présente. Au centre, la famille élargie et le noyau familial réduit est très important. «Les gitans du Nord ont un mode de vie régi par des traditions familiales «tribales» et patriarcales particulièrement fortes qui les font se sentir supérieurs aux autres gitans d'Espagne $» .{ }^{10}$ Cette structure communautaire, note encore Nancy Thede, est d'ailleurs l'une des grandes fiertés des gitans. "Les valeurs citées ont le plus souvent à voir avec le degré de solidarité interne et la qualité de vie en communauté qui en résulte. ${ }^{11}$ L'éphémère et la fragilité de la structure habitée sont contrebalancés par la cohésion des habitants entre eux. L'entraide de tous est indispensable à la survie de chacun.

Le groupe offre une protection réelle contre les aléas de l'existence, l'indigence, les accidents, la maladie, la vieillesse. Il permet de mobiliser des ressources, des forces, de l'aide, des savoirs, de l'argent chaque fois que cela est nécessaire. En même temps il exige de chacun des sacrifices, un investissement fort et personnel, une présence permanente dans les activités de la communauté, pour assurer le quotidien, contribuer aux réseaux de solidarité, participer aux fêtes, pour tenir son rang, alimenter les rumeurs, faire valoir et défendre son point de vue, régler les conflits, sauvegarder son honneur, venger les atteintes à ses proches... et cela maintient les personnes dans leur univers de dépendance les uns envers les autres, dans ce que l'on

${ }^{10}$ op. cit. p. 196.

11 Thede Nancy, Gitans et flamenco, les rythmes de l'identité, L'harmattan, Paris, 2005, p.208. 
appelle une culture de pauvreté, qui les oppose aux autres, et fait obstacle à toute perspective de promotion ou d'ascension sociale.

Les habitants vivent en cercle fermé, repliés sur eux-mêmes, dans la méfiance de toute intrusion extérieure. La structure du bidonville facilite cet isolement. L'accès à la Chabola est officieusement impossible à un non-initié ; pour y pénétrer, il faut y être accompagné de personnes qui se portent garantes de vous.

La structure même de la Chabola fait penser à celle d'un village fortifié, la disposition des habitations situées en périphérie, davantage soumises aux regards de l'autre, est plus dense qu'à l'intérieur, protégeant l'intimité de la communauté. Les maisons extérieures sont reliées entre elles par des palissades sommaires, comme pour former un rempart, elles sont naturellement plus fermées et opaques, les ouvertures étant tournées vers l'intérieur du périmètre ainsi délimité. Les parcelles sont de plus en plus perméables, au fur et à mesure que l'on s'enfonce dans le labyrinthe des rues. Les délimitations importantes ne se situent pas entre les habitations, mais davantage dans la relation du bidonville avec la ville institutionnelle. «El Salobral » est donc une enceinte à l'intérieur de laquelle, l'intimité de chacun des membres est moins recherchée que celle du groupe de gitans par rapport au reste de la société.

Le voisinage immédiat de la Chabola est essentiellement composé d'usines et de friches industrielles. «Les tsiganes (mais cela pourrait s'appliquer à la plupart des populations des bidonvilles) sont des experts dans l'exploitation de niches instables et changeantes, telles que celles qui apparaissent en cours de processus de modernisation rapide. Les gitans, comme la plupart des habitants de bidonville, concentrent leurs interventions dans les interstices non reconnus ou peu occupés par le reste de la société. ${ }^{12}$ Ils constituent « un groupe invisible dans la plupart des sociétés industrielles, et leur survie serait en partie due à cette invisibilité $» .^{13} \mathrm{Si}$ la Chabola, isolée par ses barrières périphériques, est une "enclave" à l'intérieur de la ville, elle n'en est pas moins dépendante de l'environnement sur lequel elle se fixe, survit en parasite, car les bidonvilles "se greffent» au voisinage de «foyers attractifs» tels que les zones industrielles, les entrepôts ou les aires de décharge et stockage de marchandises. En

\footnotetext{
${ }^{12}$ Thede Nancy, Gitans et flamenco, les rythmes de l'identité, L'harmattan, Paris, 2005, p.52.

${ }^{13}$ Lauwgie cité in Thede N., Gitans et flamenco, les rythmes de l'identité, L'harmattan, Paris, 2005, p.24.
} 
effet explique Nancy Thede : « les sociétés gitanes ne vivent jamais en autarcie ou même physiquement isolées. Elles vivent toujours en relation symbiotique avec une société hôte, dont elles épousent un certain nombre de pratiques et d'institutions permettant de s'y souder. $»^{14}$

Longtemps les gitans espagnols ont vécu en nomades, allant de fermes agricoles en fermes agricoles, où ils occupaient principalement des emplois saisonniers. C'est autour du rythme du travail de la terre et des veillées entre ouvriers agricoles tenus à l'écart dans les grandes latifundia que s'est développée la culture du flamenco, un des aspects fort de l'identité gitane. Les mutations économiques et sociales frappent toujours en priorité les plus précaires, les plus pauvres, les obligeant sans cesse à s'adapter à la société dominante. La mécanisation du monde agricole commence par supprimer les activités les moins qualifiées, les plus facilement automatisables, traditionnellement exercées par cette population. En Espagne, depuis 1980, plusieurs municipalités ont promulgué une loi interdisant la vente ambulante, privant les gitans d'un de leurs principaux métiers ${ }^{15}$. Sédentarisés, ils exercent maintenant essentiellement, au sein de la chaîne de recyclage des matériaux et des objets de consommation, de petits métiers de récupération de cartons et de ferrailles ou encore, quand cela reste possible, de la vente ambulante et de petits commerces...

\section{Esthétique de la Chabola}

Une famille regroupe plusieurs générations, parents, frères et sœurs, leurs conjoints et leurs enfants. Même si le lien de parenté est éloigné, la solidarité et l'entraide s'étendent à la famille élargie. La femme ne travaille généralement pas, sauf dans le cadre de la vente de fleurs, de vêtements ou de primeurs dans la rue et sur les marchés. Elle reste au foyer, s'occupe des enfants et des tâches domestiques. L'homme, absent durant la journée, investit ses activités professionnelles précaires, les combines du moment.

Les habitations sont implantées à proximité les unes des autres, avec en général des espaces intermédiaires permettant à la Chabola de se densifier progressivement. La parcelle «gitane» reprend ici le tracé de l'ancienne parcelle des jardins potagers, occupée illégalement par les populations de "San Cristobal» et rachetée «au noir» par les gitans. Elle est occupée par une famille ou un groupe d'une même

\footnotetext{
${ }^{14}$ op. cit. p.51.

${ }^{15}$ op. cit. p.26.
} 
parentèle, dans ce cas plusieurs maisons sont construites sur une même parcelle, recoupée en petits lots, avec des délimitations plus ou moins poreuses et une entrée qui peut être commune. De petits chemins piétonniers permettent aux habitations de communiquer entre elles à l'intérieur de la grande parcelle familiale. Même si l'esprit de communauté est maintenu, chaque noyau familial vit dans sa maison. Une fois mariés, les enfants prennent leur indépendance en construisant leur propre habitation sur la parcelle familiale.

La maison est généralement composée d'une pièce principale, cubique, fermée et d'un auvent attenant ouvert sur l'extérieur. Ce dernier fait fonction de seuil, d'espace de transition entre la vie privée, réservée au noyau fermé de la famille, et l'espace collectif de la rue, « salon ouvert » pour y manger, s'y reposer, se réunir. La cuisine peut gagner elle aussi l'extérieur, grâce à un évier aménagé sommairement et un foyer. Il n'est pas rare de voir le réfrigérateur et des meubles de cuisine border cet espace, jalonné de fauteuils, chaises et tables. Un miroir est souvent accroché en façade.

La partie fermée de l'habitation ne possède qu'une seule entrée donnant directement sur la pièce principale qui mesure de 10 à $30 \mathrm{~m} 2$. Elle est peu meublée : canapé, chaises et table. Un plan de travail constitue la cuisine équipée d'une plaque électrique et parfois d'un four. Les meubles sont disposés le long des parois de la pièce. Le salon se convertit en chambre pour la nuit. Une pièce plus petite peut y être ajoutée : c'est la chambre matrimoniale. Généralement, quand la taille le permet, les enfants dorment dans la chambre parentale. Ces espaces ne sont pas fermés par une porte, mais occultés par un rideau.

Les ouvertures sont réduites au strict minimum nécessaire à la circulation et à l'aération, car l'hiver, elles sont aussi source de déperdition de chaleur, de plus, elles posent des problèmes structurels, qui fragilisent l'habitation et accroissent sa perméabilité. Les fenêtres, quand il y en a, sont de taille réduite $(50 \times 50 \mathrm{~cm}$ en moyenne). L'éclairage est donc artificiel; dans la majorité des cas, une ampoule par pièce suffit pour éclairer faiblement le foyer. Bien que vivant en communauté, les habitants du bidonville doivent s'efforcer de ne pas éveiller la convoitise, car les chapardages sont facilités par la précarité de l'habitation. Il n'y a pas d'étalage de richesse, cela suffit à réduire les problèmes de vols. L'habitat est clos, sombre et sécurisant.

Il n'y pas d'eau courante et l'eau du puits n'est pas potable. Les familles achètent des cubes de mille litres d'eau, qu'ils mettent à l'extérieur. Ils peuvent être montés sur des échafaudages pour reprendre le principe du château d'eau. L'espace réservé au bain et à 
l'hygiène se situe à l'extérieur de la baraque. Il est semi fermé (les parois ne touchent ni le sol ni le plafond pour permettre une meilleure aération et l'écoulement des eaux), des rideaux de tissus protègent l'intimité. Une fosse sur laquelle vient se poser un sanitaire fait office de toilette. Il n'y a pas de douche ni de baignoire. Les habitants se lavent au seau. L'espace est relativement vaste $(4$ à $8 \mathrm{~m} 2)$, et permet d'accueillir plusieurs personnes en même temps. A cette structure de base, peuvent s'ajouter d'autres composants : un puits, un poulailler, un enclos pour l'âne et le cochon, un verger quand la taille de la parcelle le permet, un abri pour la voiture.

«Les favelas sont le résidu du résidu, le reste du reste urbain. ${ }^{16} \mathrm{Le}$ statut irrégulier du bidonville fait que celui-ci se développe avec le risque d'être détruit du jour au lendemain; les projets d'habitation sont pensés, préparés en intégrant cette donne de façon plus ou moins consciente et en la conjuguant avec les faiblesses des ressources mobilisables. L'accumulation et le stockage préalable de matériaux de récupération en est la condition. La main qui assemble les matériaux de la future habitation a une façon propre de résoudre les problèmes ; la fameuse « réserve », l'amoncellement d'éléments de récupération disparates, offre toujours la possibilité aux constructeurs de choisir en fonction de leur ingéniosité, au sein d'un panel des différentes solutions possibles. La précarité de la construction fait qu'elle n'est jamais terminée et qu'il faut toujours la consolider et l'améliorer. Une famille doit engager des travaux pour boucher une fuite d'eau ou renforcer un auvent qui menace de s'effondrer. Pour trouver les matériaux nécessaires, la vigilance du père est en alerte, de façon à repérer une planche ou tout autre élément pouvant faire l'affaire et résoudre, même temporairement, le problème. Le bâtiment est sans cesse bricolé, raccommodé, réaménagé, il se transforme au rythme de la vie de ses habitants et en fonction de leur quotidien aléatoire. À propos des favelas, Jacques Berenstein explique qu'elles ont ainsi une esthétique propre, les travaux d'isolation ou d'amélioration, réalisés avec un « degré minimal d'intuition constructive » ${ }^{17}$, sont fréquents, et donnent l'idée que la «peau» qui recouvre la maison mue. Il en résulte, conclut-il, " une sensation d'instabilité, d'un certain inconfort donnant l'impression de déséquilibre », ou plutôt, "d'un équilibre dynamique qui se déplace $» .{ }^{18}$

\footnotetext{
${ }_{17}^{16}$ idem, p.43.

${ }^{17}$ Berenstein Jacques, Esthétique des favelas, L'Harmattan, 2002, p.34.

${ }^{18}$ op. cit., p.32.
} 
Le fait que les matériaux de récupération proviennent de lieux proches traduit l'appartenance du bidonville à un environnement, un contexte qui lui fournit les matières premières nécessaires à son édification. Ici, le fait que la Chabola se situe à proximité d'une zone industrielle explique l'utilisation d'emballage tetrabrik ${ }^{\circledR}{ }^{19}$ comme membrane isolante. Ce procédé ingénieux, efficace en terme d'isolation et de protection contre la pluie est facile à se procurer et à mettre en œuvre ; si bien que la solution s'est répandue et propagée à l'ensemble des habitations. Le tetrabrik s'est donc généralisé comme matériau de base pour la construction de l'habitation. Il a par la suite été décliné selon l'inventivité et le goût de chacun. Les gitans achètent maintenant directement aux entreprises des fins de rouleaux utilisés pour la fabrication des emballages, si bien qu'on le retrouve posé à l'intérieur à la manière d'un papier peint.

Les fondations sont constituées d'une dalle de béton dans laquelle sont encastrés les poteaux de bois de la structure porteuse de la maison et de la pergola. L'espace vacant entre ces éléments est comblé par des tôles, de la terre, du film plastique, des planches de bois et par tout autre élément de récupération (panneaux d'affichage, sommiers, barres de métal...). Le toit est constitué de tôles métalliques reposant sur des poutres de bois lestées par des pneus. Le résultat final est disparate. Cependant, les finitions sont «soignées», avec une recherche de composition et de couleur, dans un projet esthétique, imperceptible au premier abord. Il est évident que ce n'est pas le souci majeur d'un jeune ménage construisant son premier «logis». Mais peu à peu, les femmes qui restent au foyer et élèvent les enfants usent d'astuce pour égailler la misère quotidienne. Qui repeint sa maison en rose, qui installe telle affiche de mode ou tel panneau publicitaire en carton sur la façade de l'habitation. Ces petits riens dessinent ainsi, au jour le jour, un paysage original ou "même le quotidien le plus dérisoire garde une trace de grandeur et de poésie spontanée. ${ }^{20}$

La maison n'est pas étudiée pour durer, mais pour tenir un certain temps, au delà duquel elle est rapiécée. La somme de chaque petit élément disparate ajouté aux autres fait que l'ensemble des cahutes crée, à l'échelle de la communauté, un patchwork à l'esthétique déconstruite, mais spécifique du lieu.

\footnotetext{
${ }^{19}$ Le tetrabrik nom commercial, fabriqué par la Maison Tetra Pak, se compose de 5 films superposés, 3 en plastique polyéthylène, 1 en aluminium, 1 en papier Kraft. Ces films sont notamment utilisés pour les emballages de packs de lait.

${ }^{20}$ Lefebvre Henri «La révolution urbaine» cité in Pinson Daniel, Usage et architecture, L'harmattan, Collection Ville et Entreprises, Paris, 1993, p.156.
} 
A l'intérieur des habitations, la doublure étanche en tetrabrik ${ }^{\circledR}$ devient une composante décorative déclinée selon les goûts de chacun; les différents motifs d'emballage de l'industrie agro-alimentaire décorent séjour et chambre. Dessins colorés, frises horizontales ou verticales, laissent imaginer l'effort déployé pour aménager un intérieur plus convivial. «Une baraque de bidonville, défend D. Pinson, a une plus grande valeur d'usage pour les déshérités qu'une maison en dur faite selon les normes les plus modernes », en effet "même dans les limites extrêmement étroites, celles des ressources dont ils disposent, les habitants des bidonvilles décident et réalisent selon leurs intentions $\gg .{ }^{21}$

\section{Bidonville et ville gentrifiée}

Les bidonvilles, que dénonçaient l'abbé Pierre et A.T.D. Quart Monde dans les années 60, étaient tant bien que mal acceptés comme des zones de parcages temporaires. Les émigrés qui les habitaient devaient passer ensuite par des cités justement appelées «de transit», parce qu'elles les préparaient et les aidaient à rejoindre la société dans laquelle ils devaient s'intégrer progressivement, en renonçant aux aspects les plus caractéristiques de leur culture d'origine. Il en a été de même pour une partie des premiers habitants de la Chabola d'El Salobral, qui ont bénéficié d'une politique de relogement et cédé la place à de nouveaux arrivants. Bien sûr, les voix étaient nombreuses pour dire que ce n'était pas aussi simple, que les moyens nécessaires étaient insuffisants, mais enfin, le projet était là. Et en Europe, la première étape au moins, de résiliation des bidonvilles, n'a pas si mal fonctionné, facilitée par la forte croissance caractéristique de ces années là.

Les bidonvilles planétaires relèvent d'une toute autre logique ; il n'y a plus de projet d'insertion ni même d'interrogation sur le devenir de ces populations; tout au plus constituent-elles un sous prolétariat pléthorique, dont les villes et les industries ne savent plus que faire... Les municipalités les abandonnent à leur sort, contraintes de les tolérer là où elles s'installent, pour autant qu'elles demeurent entre elles et ne débordent pas sur le reste de la ville. Ainsi les bidonvilles livrés à eux-mêmes, deviennent-ils des lieux de relégation ad vitam aeternam; livrés à la violence des caïds locaux, des milices, des pègres, des polices parallèles, aux mafias de toutes sortes, qui arrosent

\footnotetext{
${ }^{21}$ Pinson D., Usage et architecture, L'harmattan, Collection Ville et Entreprises, Paris, 1993, cite une étude de Turner J, datant de 1968, sur l'appropriation, p.119.
} 
les fonctionnaires locaux, prélèvent leurs taxes, font la loi et imposent, à leur façon, un semblant d'ordre.

Les populations déracinées, quand elles peuvent se retrouver en familles, en clans, en ethnies, font de la culture d'origine un ciment qui les réunit, les préserve, leur permet de développer et d'entretenir des formes de solidarité indispensable à leur survie. Leur culture d'origine leur donne un sentiment de permanence face à la précarité absolue de leurs conditions de vie. Pour donner un peu de sens et de bonheur à leur existence, les habitants retrouvent ou inventent des manifestations ethniques festives, qu'ils maintiennent envers et contre tout, mais qui font finalement obstacle à leur intégration. Et cela persiste d'autant plus, que le bidonville devient une enclave, que les liens avec l'extérieur sont faibles, que les gens travaillent au mieux la nuit, dans les marges, ou selon des rapports sociaux de relégation et de grande précarité.

A ces formes de résistances relativement traditionnelles, s'ajoute une nouvelle donne : celle des religions dans leur expression la plus dure et intégriste, celle des fondamentalistes islamistes bien sûr, mais aussi d'autres religions, notamment chrétiennes pentecôtistes. Ce sont les seules à organiser une alternative aux mafias, à proposer et à défendre un minimum d'assistance sociale et à entretenir un ensemble de structures de bienfaisance. Mais c'est aussi à la condition de durcir la relation d'altérité, par un ascétisme et une soumission aux règles les plus fondamentalistes, les plus rigoureuses de ces religions, dont les rites et les rythmes rigides imposent des formes de sociabilité qui éloignent les croyants des autres, des non croyants, et notamment aussi des nouveaux modes de vie urbains souples, évolutifs et fluides. La communauté se durcit, se construit dans une représentation violente du rapport aux autres, aux infidèles. On pense à l'islamisme fondamentaliste, mais il en est de même des pentecôtistes. Ces derniers, outre leurs pratiques extatiques afro-américaines, sont prémillénaristes ; ils croient en une guerre mondiale imminente entre le capital et le travail, persuadés (mais qui pourrait les contredire) que la vie urbaine est corrompue, injuste et impossible à réformer; à l'issue de cette guerre, et pour une période de mille ans, régnera la justice universelle du Christ. Alors qu'ils étaient très majoritairement catholiques, d'après Nancy Thede, près de $50 \%$ des gitans espagnols seraient désormais pentecôtistes ${ }^{22}$.

\footnotetext{
22 Thede Nancy, Gitans et flamenco, les rythmes de l'identité, L'harmattan, Paris, 2005 , p.36.
} 
A l'opposé, la ville postmoderne «officielle » se gentrifie même dans ses marges ${ }^{23}$. Elle se rénove, se restaure, réhabilite ses quartiers anciens, ses docks et ses friches industrielles dans le souci de redonner un peu d'épaisseur historique et d'unité esthétique à son urbanisme. Elle retrouve ou s'invente des racines, différentes selon les époques, les lieux, les modes du moment, car tout cela reste superficiel, évolutif. Elle se met en évidence, se patrimonialise. Elle se met en scène comme zone de chalandise fluide pour la consommation, les loisirs, la sociabilité, le plaisir de ses classes moyennes aisées, qui la parcourent de long en large, en réinventant les usages, se dispersant et se recomposant en tribus éphémères, pour un verre, un repas, une manifestation ou un événement. Et chacun de multiplier les appartenances, de cultiver les multiples facettes d'une identité personnelle, composite mais éphémère, qui le transforme tour à tour en badaud, en manifestant, en festivalier, en raveur, en fêtard, en congressiste, en conventionnaire, en touriste, en amateur, en spectateur, en marathonien, en supporter... Et chacun de cultiver son style avec les équipements sophistiqués, les tenues vestimentaires et les accoutrements appropriés, devenus tellement indispensables. Et chacun de courir la ville, de lieux de rendez-vous en lieux de rendezvous, avide d'en être, insatiable d'expériences nouvelles, de métissage, de cosmopolitisme, «open », ouvert à tous les vents, à toutes les influences, aux expériences les plus folles, les plus saugrenues, extatiques, exotiques, sexuelles, sensuelles, fantasmagoriques, sensibles, émouvantes, "adrénalisantes", épuisantes. A l'heure post industrielle, remarque encore le sociologue américain Rifkin, la sociabilité, les loisirs, la culture, l'échange d'informations, le sentiment d'en être, deviennent des denrées de grande consommation, réservées à ceux qui peuvent se les payer et ont les moyens de se brancher sur les réseaux formels et informels d'accès à ces multiples pratiques distinctives, dont sont irrémédiablement exclus les habitants des bidonvilles. ${ }^{24}$

Et si l'important est d'en être, c'est à condition toutefois que ces expériences, si fortes soit-elles, demeurent limitées dans le temps. Alors que l'engagement religieux à l'œuvre dans les bidonvilles est total, progressif, initiatique, mais irrémédiable et de plus en plus exigeant, ces expériences sont «flashantes", elles offrent les

\footnotetext{
${ }^{23}$ Voir sur le sujet : Bauman Zygmunt, L'amour liquide, De la fragilité des liens entre les hommes, Rodez, Le Rouergue, Chambon, 2004 ( $1^{\text {re }}$ éd. en anglais 2003). Rifkin Jeremy, L'Âge de l'accès, La Nouvelle culture du capitalisme, Paris, La Découverte, 2005 ( $1^{\text {re }}$ éd. en anglais 2000).

${ }^{24}$ Jeremy Rifkin, L'Âge de l'accès, op. cit., p.310 et suivantes.
} 
sensations les plus fortes, les plus belles émotions, mais ne durent que l'espace d'un instant et sont absolument sécurisées, elles garantissent, formellement, de revenir toujours sain et sauf, car c'est la condition sine qua non pour pouvoir passer de l'une à l'autre.

\section{Conclusion}

A l'immobilisme, à la relégation et à la précarité des habitants des bidonvilles, enfermés dans leur fondamentalisme, isolés des autres par leur culture d'origine ethnique ou d'appartenance religieuse, s'oppose radicalement la fluidité et la sécurité des nouveaux usages de la ville, la liberté et la diversité des affiliations multiples à des clubs, des cercles, des sociétés, des groupes de métissage des cultures du monde, ou du moins ce qu'il en reste.

La ville gentrifiée met en évidence sa durée, son histoire, les différentes strates de son bâti, ruines romaines, vieux centres moyenâgeux, beaux quartiers belle époque, périphérie et gares de la première mondialisation; elle réhabilite, consolide, classe, met en valeur. A l'opposé, les bidonvilles se bidonvillisent, quand les cours des maisons ou même les toits sont loués par les plus anciens habitants aux derniers arrivants ; ils sont sans bâti et n'ont d'autre histoire que la mouvance des populations qui l'habitent et s'inventent des origines fondamentalistes, ou réactivent les liens de sang.

La ville organise sa circulation toujours plus fluide, toujours plus dense, toujours plus rapide, toujours plus en mouvement, avec ses gares, ses aéroports, ses départs, ses arrivées, en périphérie desquelles viennent errer les gens des bidonvilles, avides de petits boulots, mais qui eux ne partent jamais; dans les Chabolas, même les gens du voyage se sont arrêtés... Les bidonvilles sont engorgés, impropres à toute circulation; pour leurs habitants, les seuls grands voyages possibles sont leur plus grande crainte, celui de l'expulsion par charters de rapatriement...

Les quartiers du centre ville en cours de gentrification, tout de circulation, de communication et de métissage, s'opposent, par bien des aspects, symétriquement aux bidonvilles, où viennent s'agglutiner, se sédimenter les populations marginales, à bout de force et d'errance; enclaves de non droits et de relégation pour des populations embourbées dans la précarité. Les déracinés y conservent des bribes de leur culture d'origine, qu'ils réactivent pour susciter des formes d'appartenances, pour créer du lien, de la solidarité au sein de la communauté et dresser des frontières entre eux et tous les autres, une façon de résister, de survivre, dans des conditions 
particulièrement difficiles. Ce que les sociologues des années 70 appelaient la culture de pauvreté, tend à se durcir avec la montée de l'intégrisme, qui conduit à une radicalisation de ces oppositions. Les communautés se referment, s'opposent, cultivent le communautarisme, ce qui en définitive arrange bien toutes les formes de pouvoir, celles des mafias sur le terrain des bidonvilles, celles du pouvoir urbain ailleurs, qui abandonnent aux caïds, aux mollahs, aux prêcheurs, ces zones de non-droit, sous réserve que ces derniers les contrôlent et limitent les révoltes ou les exactions à l'extérieur. Du coup, l'économie de politiques sociales et sécuritaires pourtant bien indispensables est faite. Et les autres de circuler, de voyager, de se retrouver, de faire la fête, de danser sur un volcan...

\section{Bibliographie}

Augé M., Pour une anthropologie des mondes contemporains, Champs, Paris, Editions Flammarion, 2001.

Bauman Z., L'amour liquide, De la fragilité des liens entre les hommes, Rodez, Editions Le Rouergue, Chambon, 2004 ( $1^{\text {re }}$ éd. en anglais 2003).

Berenstein J., Esthétique des favelas, Paris, Editions L'Harmattan, 2002.

Davis M., Planète Bidonvilles, Bachmann B., Les enfants d'une même agonie, Paris, Editions Ab Irato, 2005.

Rasse P., La rencontre des mondes, Diversité culturelle et communication, Paris, Editions Armand Colin, 2006

Rasse P., L'anthropologie de la communication pour étudier la rencontre des mondes, in : Communication interculturelle et diversité en Méditerranée, sous la dir. de Françoise Albertini, Éditions Dumane, 2006.

Rifkin J., L'Âge de l'accès, La Nouvelle culture du capitalisme, Paris, Editions La Découverte, 2005 ( $1{ }^{\mathrm{re}}$ éd. en anglais 2000).

Thede N., Gitans et flamenco, les rythmes de l'identité, Paris, Editions L'harmattan, 2005. 